\title{
Der Verbandsbegriff in wissenschaftlichen Publikationen. Eine Meta-Analyse zu Terminologie und Charakteristika
}

\author{
Franziska Oehmer
}

Dem Beziehungsgeflecht und Interaktionen zwischen Parlamenten und Interessenverbänden gilt die Aufmerksamkeit zahlreicher wissenschaftlicher Publikationen: So werden der Stellenwert parlamentarischer Akteure als Adressaten der Verbandsarbeit ${ }^{1}$, die personellen Verflechtungen zwischen Parlamentariern und Verbänden (innere Lobby) ${ }^{2}$ sowie der Einfluss von Verbänden im parlamentarischen Willensbildungs- und Gesetzgebungsprozess ${ }^{3}$ beleuchtet. Der Analysegegenstand Interessenverband divergiert dabei jedoch stark: Je nach Forschungsperspektive, wissenschaftlicher Disziplin und theoretischem Ansatz werden unter dem Begriff verschiedene Organisationsformen und Akteurstypen subsumiert oder ausgeschlossen. ${ }^{4}$ So werden beispielsweise Kirchen ${ }^{5}$, Kammern ${ }^{6}$, soziale Bewegungen ${ }^{7}$ oder auch Gebietskörperschaften des öffentlichen Rechts ${ }^{8}$ teilweise zu Interessenverbänden gerechnet oder als eigenständige Akteurstypen konzipiert. Unklar ist auch, inwiefern Nichtregierungsorganisationen (NROs, englisch NGOs) als Interessenverbände oder als eigenständige Akteurstypen gelten müssen. Diese wenig trennscharfen Abgrenzungen und die Vielzahl unterschiedlicher Akteursdefinitionen erschweren insbesondere die Vergleichbarkeit der empirischen Studien, die zwar mit Interessenverbänden denselben Untersuchungsgegenstand benennen, jedoch jeweils andere Organisationen mit divergierenden Handlungslogiken berücksichtigen. Dieser Mangel an einer einheitlichen Definition des zentralen Untersuchungsobjekts gilt zugleich auch als "größter Schwachpunkt"9 der Verbandsforschung.

Dabei erschwert die Vielfalt und Heterogenität an Interessenverbänden bereits selbst „eine einfache, allgemeinverbindliche Definition dieses Typus sozialer Organisationen “10.

1 Vgl. Martin Sebaldt / Alexander Straßner, Verbände in der Bundesrepublik Deutschland. Eine Einführung, Wiesbaden 2004.

2 Vgl. unter anderem Ulrich Lohmar, Das Hohe Haus. Der Bundestag und die Verfassungswirklichkeit, Stuttgart 1975; Jürgen Weber, Interessengruppen im politischen System der Bundesrepublik Deutschland, München 1977; Peter Schindler, Datenhandbuch zur Geschichte des Deutschen Bundestages 1949 bis 1999, Baden-Baden 1999.

3 Vgl. Walter Haller / Alfred Kölz, Allgemeines Staatsrecht, Basel / Genf / München 2004.

4 Vgl. Ullrich Willems / Thomas von Winter, Interessenverbände als intermediäre Organisationen. Zum Wandel ihrer Strukturen, Funktionen, Strategien und Effekte in einer veränderten Umwelt, in: dies. (Hrsg.), Interessenverbände in Deutschland, Wiesbaden 2007, S. 13 - 50, S. 19.

5 Vgl. Martin Sebaldt / Alexander Straßner, a.a.O. (Fn. 1); RolfHackenbroch, Verbände und Massenmedien. Öffentlichkeitsarbeit und ihre Resonanz in den Medien, Wiesbaden 1998.

6 Vgl. Martin Sebaldt / Alexander Straßner, a.a.O. (Fn. 1).

7 Vgl. Werner Reutter, Deutschland, in: ders. / Peter Rütters (Hrsg.), Verbände und Verbandssysteme in Westeuropa, Opladen 2001, S. 75 - 101.

8 Vgl. ebenda; Martin Sebaldt / Alexander Straßner, a.a.O. (Fn. 1).

9 Stefan Brieske, Public Relations der Verbände. Kommunikation im Schatten des Lobbying, Osnabrück 2007, S. 10. Vgl. auch Ullrich Willems / Thomas von Winter, a.a.O. (Fn. 4), S. 17; Joachim Preusse / Sarah Zielmann, Funktion und Stellenwert von PR in Interessenverbänden der Wirtschaft, in: PR Magazin, 40. Jg. (2009), H. 5, S. 63 - 68.

10 Francois Höpflinger, Verbände, in: Ulrich Klöti (Hrsg.), Handbuch Politisches System der Schweiz, Bern / Stuttgart 1984, S. 163 - 187, S. 163. 
Dementsprechend vielfältig und mit divergierenden Akzentsetzungen sind die in der Literatur vorzufindenden Begriffsbestimmungen.

In der häufig zitierten Definition eines Verbandes von Max Weber (1922) wird vor allem auf den Organisationsaspekt und die Binnenregulierung von Interessenverbänden verwiesen:

„Verband soll eine nach außen regulierend beschränkte oder geschlossene soziale Beziehung dann heißen, wenn die Innehaltung ihrer Ordnung garantiert wird durch das eigene auf deren Durchführung eingestellte Verhalten bestimmter Menschen: eines Leiters und, eventuell, eines Verwaltungsstabes, der gegebenenfalls normalerweise zugleich Vertretungsgewalt hat. "11

Hingegen verweist Hans-Peter Ullmann (1988) neben der Organisation und dem Interessenvertretungsanspruch auch auf die Vermittlungsleistung von Interessenverbänden als intermediäre Akteure:

„Sie [die Interessenverbände] organisieren Interessen und vertreten diese gegenüber anderen sozialen Gruppen oder den Organen der politischen Willensbildung. Damit gehören die Verbände zu jenem weiten intermediären Bereich, der sich zwischen der Lebenswelt des einzelnen auf der einen und den politischen Institutionen auf der anderen Seite auftut. "12

Andere Autoren verweisen zudem auf die Dauerhaftigkeit von Interessenverbänden ${ }^{13}$ und die starke Fokussierung auf wenige Gegenstände wie beispielsweise auf Arbeitnehmerrechte oder auf den Umweltschutz ${ }^{14}$ als bestimmende Merkmale.

Die Heterogenität der Verbandsdefinitionen spiegelt sich auch in der Vielzahl der divergierenden Bezeichnungen, die zum Teil synonym, „teils über- oder unterordnend“15 verwendet werden: Interessenverband, Interessengruppe, Interessenorganisation, organisierte Interessen, pressure group, lobby. ${ }^{16}$ Die Begriffe pressure group und lobby verengen jedoch die Organisationstätigkeit auf jeweils eine bestimmte Methode der politischen Einflussnahme: Während ersterer vor allem auf die Druck ausübenden Aktivitäten wie beispielsweise Streiks

11 Max Weber, Soziologische Grundbegriffe, Tübingen 1984, S. 26.

12 Hans-Peter Ullmann, Interessenverbände in Deutschland, Frankfurt am Main 1988, S. 9.

13 Vgl. Martin Sebaldt / Alexander Straßner, a.a.O. (Fn. 1); Wolfgang Beutel, Verbände: Interessenorganisation und Gemeinwohl - ein Konflikt?, in: Wichard Woyke (Hrsg.), Interessenvermittlung durch Verbände, Schwalbach 2004, S. 80 - 90; Peter Raschke, Vereine und Verbände. Zur Organisation von Interessen in der Bundesrepublik Deutschland, München 1978; Herbert Krüger, Die Verbände, in: Rudolf Steinberg (Hrsg.), Staat und Verbände. Zur Theorie der Interessenverbände in der Industriegesellschaft, Darmstadt 1985, S. 118 - 142; Beatrice Dernbach, Berufsfeld Verbände, in: Günter Bentele / Romy Fröhlich / Peter Szyszka (Hrsg.), Handbuch der Public Relations, Wissenschaftliche Grundlagen und berufliches Handeln, Wiesbaden 2008, S. 468 - 481; Ullrich Willems / Thomas von Winter, a.a.O. (Fn. 4); Bernhard Thibaut, Interessengruppen/Interessenverbände, in: Dieter Nohlen / Rainer-OlafSchultze (Hrsg.), Lexikon der Politikwissenschaft. Theorien, Methoden, Begriffe, Band I, München 2002, S. 380; Dieter Witt / Gernot Seufert / Hannes Emberger, Typologisierung und Eigenarten von Verbände, in: Zeitschrift für öffentliche und gemeinnützige Unternehmen (ZögU), 19. Jg. (1996), H. 4, S. $414-427$.

14 Vgl. Bernhard Schreyer, Grundkurs Politikwissenschaft: Studium der politischen Systeme. Eine studienorientierte Einführung, Wiesbaden 2002; Bernhard Thibaut, a.a.O. (Fn. 13); Joachim Jens Hesse / Thomas Ellwein, Das Regierungssystem der Bundesrepublik Deutschland, Opladen 1987.

15 Peter Raschke, a.a.O. (Fn. 13), S. 20.

16 Vgl. Martin Sebaldt / Alexander Straßner, a.a.O. (Fn. 1), S. 19; Ullrich Willems / Thomas von Winter, a.a.O. (Fn. 4), S. 21; Otfried Jarren / Patrick Donges, Politische Kommunikation in der Mediengesellschaft. Eine Einführung, Wiesbaden 2006, S. 132; Ulrich von Alemann, Verbände und Staat. Vom Pluralismus zum Korporatismus; Analysen, Positionen, Dokumente, Opladen 1979, S. 29. 
und Demonstrationen einer Organisation verweist, sieht letzterer die Hauptaufgabe in der direkten Beeinflussung der parlamentarischen Gesetzgebung ${ }^{17}$, die jedoch nicht nur von Verbänden, sondern auch von anderen Organisationen oder auch Personen intendiert sein kann. ${ }^{18}$ Im französischen und italienischen Wissenschaftsdiskurs hat sich vor allem die Übersetzung von pressure group als groupes de pression und gruppi di pressione durchgesetzt. ${ }^{19}$ In der wissenschaftlichen Terminologie im deutschsprachigen Raum herrschen jedoch vornehmlich die Begriffe Interessengruppen oder Interessenverbände vor. ${ }^{20}$

Der Vielfalt an wissenschaftlichen Termini stehen zusätzlich ebenso zahlreiche Selbstbezeichnungen der Organisationen gegenüber: Sie firmieren unter Namen wie Aktion, Arbeitsgemeinschaft, Club, Gesellschaft, Gemeinschaft, Gilde, Liga, Kreuz, Rat, Verein, etc. Die Namenswahl wird dabei von jeder Vereinigung selbst und frei von Vorgaben durchgeführt. Dennoch lassen sich, wie Peter Raschke in einer Analyse aller Interessenverbände in Frankfurt am Main feststellt, einige bestimmte Namenspräferenzen der verschiedenen Verbandstypen erkennen: „Die Wirtschafts-, Berufs- und Sozialanspruchsvereinigungen bevorzugten ,Verband', die Verbrauchervereinigungen nannten sich ,Verein', die Sport- und Hobbyvereinigungen hießen ,Klub' oder ,Verein', und die wissenschaftlichen Vereinigungen hießen ,Gesellschaften. “'21

Ziel der vorliegenden Studie ist es anhand einer Inhaltsanalyse der zentralen Publikationen der Politik-, Rechts-, und Kommunikationswissenschaft sowie der Soziologie ${ }^{22}$ im deutschsprachigen Raum ${ }^{23}$ die zentralen Bezeichnungen und Definitionselemente des Akteurstypes Interessenverband zu identifizieren. Auf der Basis der so gewonnenen Daten soll eine umfassende Definition von Interessenverbänden entwickelt und zur trennscharfen Abgrenzung von anderen Akteurstypen nutzbar gemacht werden.

\section{Meta-Analyse wissenschaftlicher Publikationen}

Zur Identifikation der im wissenschaftlichen Kontext verwendeten Terminologie und Definitionselemente zur Beschreibung von Interessengruppen wurde das Verfahren der MetaAnalyse, mit dem „die Ergebnisse verschiedener Untersuchungen mit gemeinsamer Thematik zusammengefasst werden, um so einen Überblick über den aktuellen Stand der Forschung zu gewinnen "24, verwandt. Dabei wird auf das Verfahren des propositionalen Inventars rekurriert, das Everett M. Rogers als „some type of content analysis for reviewing and combining verbal conclusions from a number of primary researchers" ${ }^{25}$ definiert. Charakteristik

17 Vgl. Martin Sebaldt / Alexander Straßner, a.a.O. (Fn. 1), S. 19.

18 Vgl. Herbert Schneider, Die Interessenverbände, München 1979, S. 9.

19 Vgl. Klaus von Beyme, Interessengruppen in der Demokratie, München 1980, S. 10.

20 Vgl. Günter Triesch / Wolfgang Ockenfels, Interessenverbände in Deutschland. Ihr Einfluß in Politik, Wirtschaft und Gesellschaft, München 1995, S. 15; Klaus von Beyme, Gewerkschaften und Arbeitsbeziehungen in kapitalistischen Ländern, München 1977, S. 10.

21 Peter Raschke, a.a.O. (Fn. 13), S. 91.

22 Zum Teil wurden auch Werke der Rechtswissenschaft in die Analyse einbezogen.

23 In der Analyse wurden nur Publikationen aus dem deutschsprachigen Raum berücksichtigt, da so kulturell und sprachlich bedingte Verzerrungen in der Merkmalsattributierung weitgehend ausgeschlossen werden konnten.

24 Jürgen Bortz / Nicola Döring, Forschungsmethoden und Evaluation, Berlin 1995, S. 589.

25 Everett M. Rogers, Methodology for Meta-Research, ICA Conference, Minneapolis 1981, S. 10. 
des propositionalen Inventars ist es, den Prozess und die Kriterien der Publikationenanalyse transparent und nachvollziehbar zu machen. ${ }^{26}$

Analyseeinheit und Suchverfahren

Die Analyseeinheit der vorliegenden Studie sind wissenschaftliche Publikationen. Dabei wurde sowohl wissenschaftliche Literatur zum politischen System und dessen Akteuren im Allgemeinen als auch zur Verbandsforschung im Speziellen berücksichtigt. Untersucht wurden dabei sowohl Lexika und Grundlagenwerke des jeweiligen Faches als auch empirische Studien oder theoretische Abhandlungen, die sich Interessenverbänden widmeten. Die relevanten Publikationen wurden in einem ersten explorativen Verfahren anhand von Recherchen in Bibliothekskatalogen und themenspezifischen Bibliografien ${ }^{27}$ ermittelt und anschließend in einer Art Schneeballverfahren durch Literaturverweise in den vorgefundenen Werken ergänzt. Mehrere Publikationen desselben Autors wurden - falls keine neuen Aspekte zur Begriffsbestimmung vorkamen - nur einmal in die Analyse aufgenommen. In diesem Falle wurde jeweils die älteste oder die älteste verfügbare Publikation oder Auflage berücksichtigt.

\section{Ergebnisse}

Insgesamt wurden Begriffsbestimmungen in 32 Publikationen verschiedener Autoren ermittelt und untersucht. Auch wenn diese Fallzahl in Anbetracht der vielen Veröffentlichungen zur Verbandsthematik gering erscheint, ist sie mit Verweis auf das häufige Fehlen konkreter Definitionen beziehungsweise auf Bezüge zu bestehenden Verbandsdefinitionen in den Werken nachvollziehbar. Mehr als die Hälfte der analysierten Literatur (63 Prozent; n = 20) verfolgte eine politikwissenschaftliche Perspektive. ${ }^{28}$ Rund 13 Prozent $(n=4)$ der Publikationen wurden von Soziologen, rund zehn Prozent ( $\mathrm{n}=3$ ) von Kommunikationswissenschaftlern verfasst. Nur zwei Werke wiesen einen rechtswissenschaftlichen Blickwinkel auf (rund sechs Prozent). Fast zehn Prozent $(\mathrm{n}=3)$ entfielen auf Autoren anderer wissenschaftlicher Disziplinen, die in der Analyse unter Sonstiges gefasst wurden. Dazu zählen Fachgebiete wie die Betriebswirtschaftslehre oder die Pädagogik.

Die untersuchten Publikationen erschienen in einem Zeitraum von rund 50 Jahren - mit dem ersten Werk aus dem Jahr 1960 und der aktuellsten Literatur aus dem Jahr 2008. Die meisten sind im Zeitraum von 2000 bis 2007 ( $n=14$ ) erschienen, was sich sicherlich mit der leichten Verfüg- und Auffindbarkeit von zeitnah publizierten Werken erklären lässt.

26 Vgl. Heinz Bonfadelli / Werner A. Meier, Meta-Forschung in der Publizistikwissenschaft. Zur Problematik der Synthese von empirischer Forschung, in: Rundfunk und Fernsehen, 32. Jg. (1984), H. 3, S. $537-550$.

27 Vgl. Kurt P. Tudyka / Juliane Tudyka, Verbände. Geschichte, Theorie, Funktion - ein bibliographisch-systematischer Versuch, Frankfurt am Main 1973.

28 Die Zuordnung der Publikationen zu einem Fachgebiet erfolgte nach der Untersuchungsperspektive des Werkes und falls diese nicht eindeutig erkennbar war, anhand des wissenschaftlichen Hintergrundes des / der Autoren. 


\subsection{Verbandsbezeichnungen}

Wie eingangs dargelegt bestimmen zahlreiche divergierende Bezeichnungen des Akteurstyps Verband den wissenschaftlichen und gesellschaftlichen Diskurs. Die hier zugrunde liegenden 32 Publikationen wurden daher auf die verwendeten Bezeichnungen untersucht. Da zumeist keine einheitliche Verwendung eines Begriffes erfolgte, wurde bei Monografien, Aufsätzen in Sammelwerken und Artikeln der im Titel verwendete Begriff als ausschlaggebende Begriffsbezeichnung gewertet. Falls dort keine Begriffsbezeichnung erkennbar war, wurde der im Text zur Definition des Akteurstyps verwendete Begriff zugrunde gelegt. In Lexika wurde das verwendete Stichwort in die Analyse mit einbezogen.

Mit rund 44 Prozent $(\mathrm{n}=14$ ) wurde in den analysierten Publikationen vor allem auf den Begriff „Verband“ zurückgegriffen. Zu rund einem Viertel wurde die Bezeichnung „Interessenverband“ ( $n=8)$ gewählt. Beide verweisen vor allem auf den Organisationscharakter von Verbänden. ${ }^{29}$ „Interessengruppe“ wurde in 19 Prozent $(\mathrm{n}=6)$ verwandt. In neun Prozent (n = 3) der Fälle wurde der Akteurstyp mit der Bezeichnung „organisierte Interessen“ betitelt, nur einmal (3,2 Prozent) als „Interessenorganisation“. Auffallend ist, dass sich keine der untersuchten Publikationen des Begriffs der pressure group oder der Lobbygruppe bediente. Das ist möglicherweise zum einen darauf zurückzuführen, dass die analysierte Literatur im deutschsprachigen Raum veröffentlicht wurde und zum anderen darauf, dass diese Begriffe - wie eingangs dargelegt - die Verbandstätigkeit auf Druck ausübende oder die politische Arbeit beeinflussende Tätigkeiten reduzieren und damit dem Anspruch wissenschaftlicher Literatur auf umfassende Erklärungskraft nicht gerecht werden. Signifikante Unterschiede in der Begriffswahl im Zeit-, oder Disziplinenvergleich konnten nicht festgestellt werden. Vor allem die Tatsache, dass es zu keinen begrifflichen Vorlieben nach Disziplin kommt, nährt die Vermutung, dass es sich bei diesem Akteurstyp um ein interdisziplinäres Untersuchungsobjekt handelt, dessen Analyse die Beachtung von Forschungserkenntnissen aller Disziplinen notwendig macht und daher auch begriffliche Adaptionsprozesse mit sich bringt.

\subsection{Konstituierende Merkmale}

Nach der Lektüre von rund 15 Prozent der untersuchten Publikationen konnten anhand der darin vorgefundenen Merkmale deduktiv Kategorien mit Charakteristika für die Inhaltsanalyse entwickelt werden, die zur Untersuchung aller - inklusive der zur Kategorienbildung genutzten - Veröffentlichungen verwandt wurden. Folgende Kategorien konnten für die Bestimmung von Interessenverbänden identifiziert werden:

Die Merkmale, die in den Definitionsbestimmungen enthalten waren, wurden entsprechend der Angaben in den Erläuterungen (vgl. Tabelle 1) den einzelnen Kategorien zugeordnet. Konnte in den Begriffsbestimmungen eine Kategorie nicht vorgefunden werden, wurde sie entsprechend als nicht vorhanden codiert.

Analysiert wurden sowohl explizite Definitionen in Lexika und Grundlagenwerken des jeweiligen Faches als auch die einer Studie oder theoretischen Abhandlung zugrunde liegenden geäußerten Annahmen über das Forschungsobjekt „Interessenverband“. In umfangreichen Monografien wurden nur die Merkmalszuschreibungen erfasst, die direkt in einem

29 Vgl. Martin Sebaldt / Alexander Straßner, a.a.O. (Fn. 1), S. 23. 


\begin{tabular}{|l|l|}
\hline Tabelle 1: Kategorien der Inhaltsanalyse \\
\hline Kategorie & Erläuterung \\
\hline Einflusslogik & $\begin{array}{l}\text { beinhaltet alle Aussagen über Außenaktivitäten eines Verbandes; Streben } \\
\text { nach politischer und gesellschaftlicher Einflussnahme; Interessendurchset- } \\
\text { zung }\end{array}$ \\
\hline Mitgliederlogik & $\begin{array}{l}\text { beinhaltet alle Aussagen über Interessenaggregation und -realisierung für } \\
\text { Mitglieder; Folgen einer Mitgliederlogik etc. }\end{array}$ \\
\hline Organisation & $\begin{array}{l}\text { beinhaltet alle Aussagen über Strukturen, Ressourcen und Regeln eines Ver- } \\
\text { bandes; oder nur die Verwendung der Wörter: Organisation, organisiert }\end{array}$ \\
\hline Freiwilligkeit & $\begin{array}{l}\text { beinhaltet alle Aussagen über die freiwillige Mitgliedschaft in einem Interes- } \\
\text { senverband }\end{array}$ \\
\hline Intermediäre & $\begin{array}{l}\text { beinhaltet alle Aussagen über Verbände als Bindeglied zwischen politischen } \\
\text { Institutionen und den Bürgern; oder nur Verwendung des Begriffs interme- } \\
\text { diär }\end{array}$ \\
\hline Dauer & beinhaltet alle Aussagen zur Langlebigkeit eines Interessenverbandes \\
\hline Nichtstaatlichkeit & $\begin{array}{l}\text { beinhaltet alle Aussagen über die Unabhängigkeit von staatlichen Eingriffen } \\
\text { / Einflüssen }\end{array}$ \\
\hline Profitwiderstreben & $\begin{array}{l}\text { beinhaltet alle Aussagen über Interessenverbände als Non-Profit-Organisa- } \\
\text { tionen }\end{array}$ \\
\hline Quelle: Eigene Zusammenstellung. \\
\hline
\end{tabular}

Begriffsbestimmungs- oder Einführungskapitel vorkamen, da hier, so die Annahme, die aus Sicht des jeweiligen Autors relevantesten Charakteristika genannt wurden. Folgende Merkmale wurden in den untersuchten Publikationen zur Bestimmung des Akteurstypes vorgefunden, wobei die Reihenfolge der Aufzählung die Häufigkeit der Merkmalsnennungen widerspiegelt.

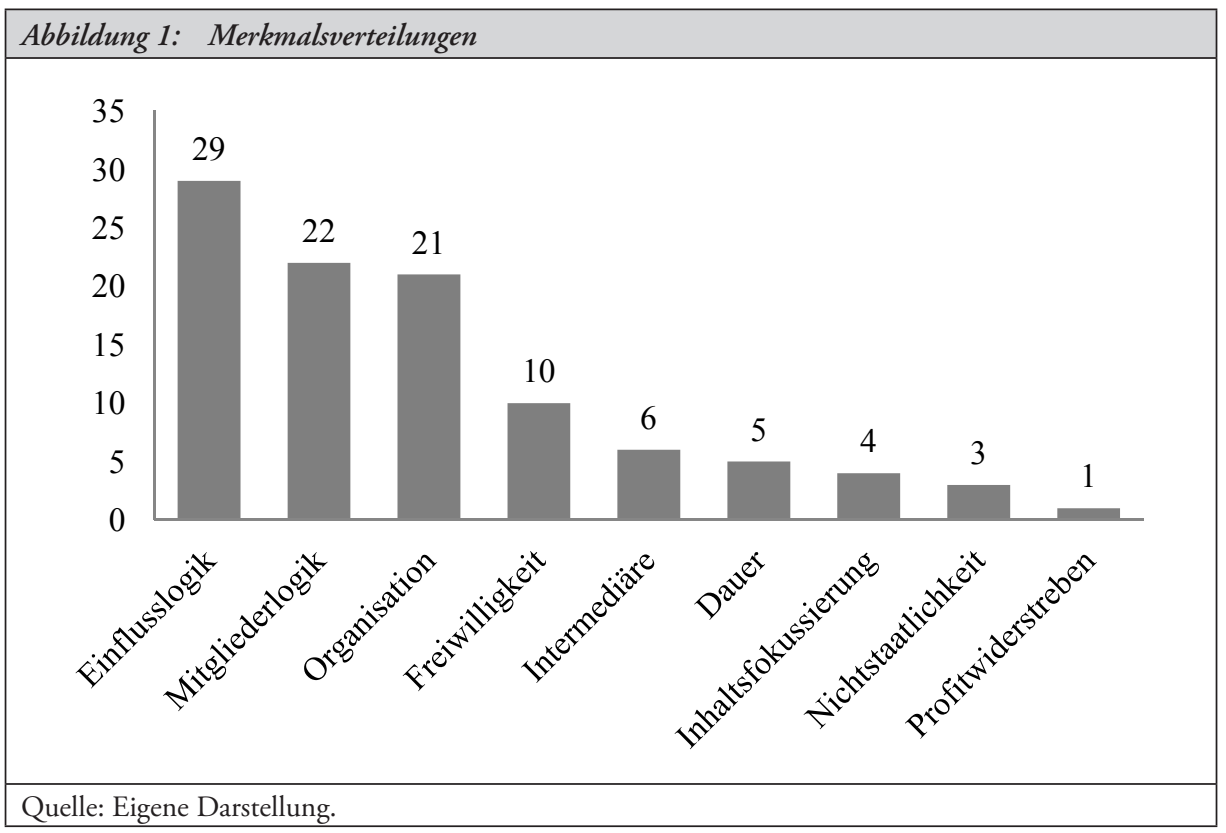


(1) Die Einflusslogik beschreibt die Außenaktivitäten eines Verbandes und damit das Streben eines Verbandes, seine Interessen im politischen, gesellschaftlichen und öffentlichen Raum sichtbar zu machen und durchzusetzen. In 28 der 32 Publikationen wurde dieses Merkmal genannt und damit diesem die größte Wichtigkeit beigemessen. Hierunter wurden Aussagen subsumiert wie: Verbände bringen Interessen „durch Mitwirkung in und Einwirkung auf Regierung, Parlament, Parteien und Öffentlichkeit im politischen Willensbildungsund Entscheidungsprozess zur Geltung “30 oder: Verbände sind Gruppen „mit dem Zweck nach außen gemeinsame Interessen zu artikulieren und direkt oder indirekt auf politische Entscheidungsprozesse Einfluss zu nehmen [... $]^{\text {"31. }}$.

(2) Ebenso viel Aufmerksamkeit $(\mathrm{n}=22)$ kommt in der Literatur dem Merkmal Mitgliederlogik zu, das auf den Vorgang der Interessenaggregation und -realisierung von Mitgliedern abzielt; Sätze wie: „Ziel des Verbandes“ sei es, den „Interessen und Forderungen der Mitglieder nach (zu)kommen “32 fielen in diese Kategorie. Auch Aussagen zu Dienstleistungen und speziellen Angeboten für eigene Mitglieder ${ }^{33}$ wurden dazugezählt.

(3) Das Merkmal Organisation $(\mathrm{n}=21)$ beschreibt das Vorhandensein von Strukturen und Ressourcen, die das Funktionieren des Verbandes sicherstellen. Auf dieses Kriterium wurde entweder durch das explizite Nennen des Organisationsbegriffes verwiesen (zum Beispiel Verbände sind „organisiert“34) oder durch den Hinweis auf feste Organisationsstrukturen mit einer Leitungsebene und fester Binnenstruktur. ${ }^{35}$

(4) Deutlich seltener $(\mathrm{n}=10)$ wird auf das Merkmal Freiwilligkeit in der Literatur eingegangen. Dieses Charakteristikum beschreibt den Umstand, dass die Mitgliedschaft freiwillig erfolgen sollte.

(5) Dass Interessenverbände nicht nur die Interessen ihrer Mitglieder im politischen Raum durchsetzen, sondern auch eine Vermittlungsleistung vom politischen System in die Alltagswelt erbringen, beschreibt das Merkmal Intermediäre $(\mathrm{n}=6)$. Es wird vor allem in Äußerungen deutlich wie: „Verbände stellen für politische Entscheidungsträger eine Verbindung zu gesellschaftlichen Teilbereichen her“36 oder: die „Interessenverbände beteiligen sich an der Umsetzung der Ergebnisse des politischen Entscheidungsprozess “ “37.

(6) Verbände zeichnen sich ebenso durch eine dauerhafte, nicht auf wenige Wochen oder Monate beschränkte Tätigkeit (Merkmal Dauer; n = 5) und durch eine Inhaltsfokussierung - eine Beschränkung auf wenige inhaltliche Themenkomplexe wie den Umweltschutz oder Interessen der Autofahrer - $(\mathrm{n}=4)$ aus.

30 Peter Massing, Interessengruppen, in: Dieter Nohlen / Rainer-OlafSchultze (Hrsg.), Pipers Wörterbuch zur Politik, Bd. 1: Politikwissenschaft, München 1989, S. 388.

31 Bernhard Thibaut, a.a.O. (Fn. 13).

32 Klaus Armingeon, Die politische Rolle der Verbände in modernen Demokratien. Fünf Thesen, in: Otfried Jarren / Dominik Lachenmeier / Adrian Steiner (Hrsg.), Entgrenzte Demokratie?, Herausforderungen für die politische Interessenvermittlung, Baden-Baden 2007, S. 107 - 122, S. 107.

33 Vgl. Ullrich Willems / Thomas von Winter, a.a.O. (Fn. 4), S. 13.

34 Bernhard Thibaut, a.a.O. (Fn. 13).

35 Vgl. Max Weber, a.a.O. (Fn. 11).

36 Sigrid Koch-Baumgarten, Verbände und Medien - „Widerspenstiges“ in der Debatte um die Mediendemokratie, in: Peter Massing (Hrsg.), Mediendemokratie. Eine Einführung, Schwalbach 2004, S. 67 - 93, S. 70.

37 Ullrich Willems / Thomas von Winter, a.a.O. (Fn. 4), S. 13. 
(7) Am seltensten wurden die Merkmale Nichtstaatlichkeit, also die finanzielle, personelle und entscheidungsgebundene Unabhängigkeit von staatlichen Instanzen, ( $n=3)$ und das nicht auf Gewinnmaximierung ausgerichtete Handeln der Interessenverbände - hier als Profitwiderstreben bezeichnet - $(\mathrm{n}=1)$ genannt.

Ähnlich wie bei den Begriffsbezeichnungen lassen sich auch bei den Merkmalszuschreibungen keine klaren Präferenzen nach Disziplinen ausmachen. Da die Mehrheit der Definitionen in Publikationen der Politikwissenschaft identifiziert worden sind, ist es wenig überraschend, dass dort auch die meisten Merkmalszuschreibungen zugeordnet worden sind. Auffällig ist jedoch, dass sich soziologische Literatur auf die Merkmale Einfusslogik, Mitgliederlogik, Organisation und Intermediär beschränken, während Inhaltsfokussierung und Nichtstaatlichkeit nur von politikwissenschaftlichen Autoren genannt wurden.

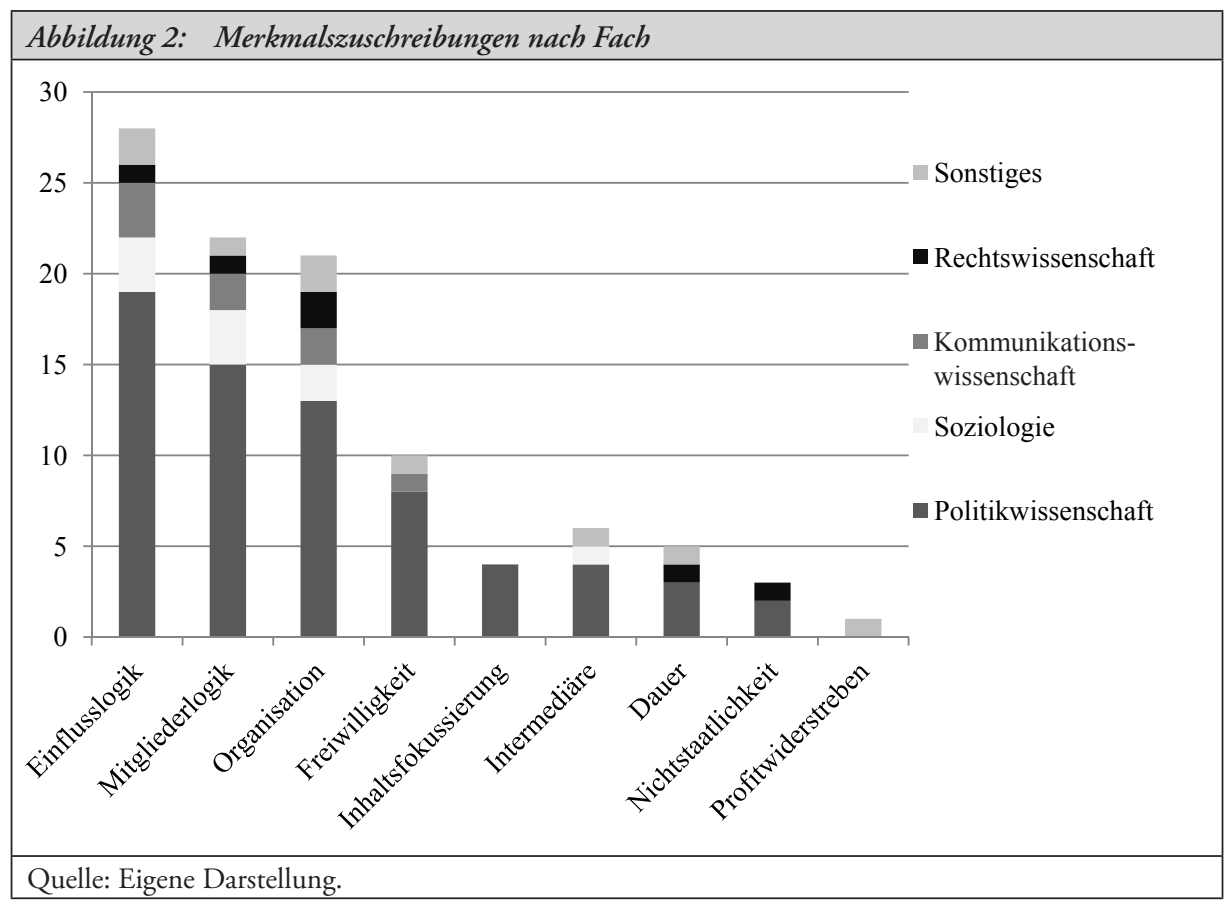

Nicht jedes der Merkmale ist jedoch in der Literatur unumstritten. So kann auch von einem Verband gesprochen werden, wenn ein Stab von hauptamtlich Tätigen nicht von formell registrierten Mitgliedern, sondern auch von Personen in Form von regelmäßigen Spenden oder Engagement unterstützt werden. ${ }^{38}$ Zudem zielen nicht alle Interessenverbände ausschließlich auf die Durchsetzung der Mitgliederinteressen im engen Sinne ab. Wohlfahrtsverbände, aber auch entwicklungs- oder umweltpolitische Interessengruppen engagieren sich für die Realisierung von Anliegen, die der Gemeinschaft oder zumindest nicht nur den eigenen Mitgliedern zugute kommen sollen. 


\section{Definition und Abgrenzung des Akteurs Interessenverband}

\subsection{Definition}

Nimmt man die erwähnten Charakteristika - unabhängig von der Häufigkeit - als Grundlage für eine umfassende Definitionsbestimmung, sind Verbände auf Dauer und auf freiwilliger Mitgliedschaft konzipierte, nichtstaatliche Non-Profit-Organisationen, die versuchen, die auf wenige Issues konzentrierten Interessen ihrer Mitglieder zu aggregieren, im politischöffentlichen Raum zu artikulieren und durchzusetzen sowie relevante Entscheidungen und Informationen des politischen Systems an ihre Mitglieder zu vermitteln.

\subsection{Abgrenzung zu anderen Akteurstypen}

Die anhand der Inhaltsanalyse identifizierten Merkmale eines Interessenverbandes dienen nicht nur einer umfangreichen Begriffsbestimmung, sondern können vor allem zur Abgrenzung von anderen Akteurstypen nutzbar gemacht werden.

Von Parteien unterscheiden sich Interessenverbände vor allem durch die Charakteristika der Nichtstaatlichkeit und Inhaltsfokussierung ${ }^{39}$ : Parteien streben in der Regel, anders als Interessenverbände, eine Regierungsverantwortung $\mathrm{an}^{40}$ und versuchen, ein breites thematisches Spektrum abzudecken. ${ }^{41}$ Zudem wird Parteien durch Artikel 21 GG das Mitwirken am politischen Willensbildungsprozess und am demokratischen Aufbau zugewiesen. ${ }^{42}$ Hierfür können sie auf eigens dafür rekrutiertes Personal zurückgreifen. ${ }^{43}$

Von sozialen Bewegungen unterscheiden sich Interessenverbände vor allem durch ihre dauerhaften Strukturen und Ressourcen. Sozialen Bewegungen fehlt es meist an interner Kompetenzaufteilung, verbindlichen Regeln der Entscheidungsfindung, Rollenspezifikationen und Mitgliedern. ${ }^{44}$ Sie besitzen somit „sozial, organisatorisch und geographisch - höchst unscharfe Ränder“ 45 . Soziale Bewegungen gelten meist als Vorläufer von Nichtregierungsorganisation, die sich anhand von drei Merkmalen klassifizieren lassen: Nicht-Staatlichkeit, Nicht-Kommerzialität und Organisation. ${ }^{46}$ All diese Merkmale können auch für privatrechtlich organisierte Interessenverbände zutreffen. Abgrenzungsversuche müssen daher als

39 Vgl. Theo Schiller, Verbände und Parteien, in: Thomas von Winter / Ullrich Willems (Hrsg.), a.a.O. (Fn. 4), S. 439 - 464, S. 440.

40 Vgl. Ursula Birsl, Was sind Interessenverbände? Definitionen, Abgrenzungen und Handlungsfelder, Technische Universität Berlin 2006, S. 23; Herbert Schneider, a.a.O. (Fn. 18), S. 11; Joachim Jens Hesse / Thomas Ellwein, a.a.O. (Fn. 14), S. 159.

41 Vgl. Bernhard Thibaut, a.a.O. (Fn. 13).

42 Vgl. Herbert Schneider, a.a.O. (Fn. 18), S. 11.

43 Vgl. Martin Sebaldt / Alexander Straßner, a.a.O. (Fn. 1), S. 22.

44 Vgl. Dieter Rucht, Parteien, Verbände und Bewegungen als Systeme politischer Interessenvermittlung, in: Oskar Niedermayer / Richard Stöss (Hrsg.), Stand und Perspektiven der Parteienforschung in Deutschland, Opladen 1993, S. 251 - 275, S. 263; ders., Soziale Bewegung, in: Dieter Nohlen / Rainer-Olaf Schultze (Hrsg.), a.a.O. (Fn. 13), Bd. 2, S. 868 - 871; Otfried Jarren / Patrick Donges, a.a.O. (Fn. 16); Francois Höpflinger, a.a.O. (Fn. 10), S. 164.

45 Dieter Rucht, Parteien, Verbände und Bewegungen als Systeme politischer Interessenvermittlung, a.a.O. (Fn. 44), S. 264.

46 Vgl. Kathrin Voss, Öffentlichkeitsarbeit von Nichtregierungsorganisationen, Wiesbaden 2007. 
wenig trennscharf und für die empirische Untersuchung als kaum fruchtbar bezeichnet werden. Christiane Frantz' zufolge divergieren sie von Interessenverbänden insofern, als sie eine andere Logik der Interessenvertretung verfolgen: Während sich Verbände überwiegend um die Durchsetzung der Mitgliederinteressen bemühen, stehen für NROs vor allem auch Anliegen von Personen oder Personengruppen, die nicht zu ihrer eigenen Klientel zählen, im Vordergrund. ${ }^{47}$ Mit Verweis auf die Aktivitäten von Verbänden aus dem karitativen, sozialen oder ökologischen Bereich, die ebenfalls nicht nur den Mitgliederbedürfnissen zur Durchsetzung verhelfen wollen, ist auch dieses Unterscheidungsmerkmal in Bezug auf seine Tragfähigkeit anzuzweifeln. Dass Nichtregierungsorganisationen im Gegensatz zu klassischen Verbänden über den Nationalstaat hinweg agieren, ist ebenso mit Blick auf die zunehmend auch europäisch oder international tätigen Interessenverbände und mit Verweis auf nur lokal oder regional arbeitende Nichtregierungsorganisationen als Abgrenzungsmerkmal wenig nützlich.

Ebenso wenig wie soziale Bewegungen konstituieren sich auch Stiftungen nicht aus Mitgliedern, sondern beschränken sich in der Regel auf Förderer und Unterstützer. ${ }^{48}$ Obwohl im juristischen Sinne zwischen Verein und Verband kein Unterschied gemacht wird ${ }^{49}$ und auch viele Verbände unter dem Namen Verein firmieren, lassen sich im wissenschaftlichen Diskurs beide Akteurstypen anhand des Merkmals der Einflusslogik voneinander abgrenzen. Klaus Armingeon begreift Vereine als „Organisationen, deren Ziele sich vordringlich auf die Leistungen im Zusammenspiel der Mitglieder und auf deren Geselligkeit ausrichten "50. Sie sind demnach überwiegend im Freizeitbereich und meist ohne politischen Hintergrund aktiv. ${ }^{51}$ Ähnlich argumentiert Stefan Brieske, wenn er nach außen gerichtete Aktivitäten und Zielsetzungen als Charakteristikum von Interessenverbänden und nach innen orientiertes Handeln als das von Vereinen konzipiert. ${ }^{52}$ Peter Raschke greift zur Unterscheidung von Vereinen und Verbänden auf die unterschiedlichen Formen der Interessenwahrnehmung zurück: Verbände vertreten überwiegend Interessen, die nur mittelbar über Dritte (politischen Institutionen etc.) realisiert werden können, Vereine hingegen verfolgen vornehmlich Interessen, die von der Vereinigung selber durchgesetzt werden können (Sport, Geselligkeit, Dienstleistungen). ${ }^{53}$ Eine Zuordnung als Interessenverband oder Verein ist jedoch nicht starr und unabänderlich: Sobald die alleinige Innenorientierung eines Vereins aufgegeben wird und auch der Versuch unternommen wird, Einfluss im politisch-gesellschaftlichen Raum geltend zu machen, kann er unter dem Akteurstyp Interessenverband subsumiert werden. ${ }^{54}$ „Selbst der unscheinbarste Verein, der sich heute ausschließlich mit Schafkopf- oder Kegel-

47 Vgl. Christiane Frantz, NGOs als transnationale Interessenvertreter und Agenda Setter, in: Otfried Jarren / Dominik Lachenmeier I Adrian Steiner (Hrsg.), Entgrenzte Demokratie? Herausforderungen für die politische Interessenvermittlung, Baden-Baden 2007, S. 181 - 197, S. 184.

48 Vgl. Dieter Witt / Gernot Seufert / Hannes Emberger, a.a.O. (Fn. 13), S. 418

49 Vgl. Frank Pilz / Heike Ortwein, Das politische System Deutschlands. Systemintegrierende Einführung in das Regierungs-, Wirtschafts- und Sozialsystem, München 2000, S. 293.

50 Klaus Armingeon, a.a.O. (Fn. 32), S. 108

51 Vgl. Martin Sebaldt / Alexander Straßner, Klassiker der Verbändeforschung, Wiesbaden 2006, S. 23; Francois Höpflinger, a.a.O. (Fn. 10), S. 164.

52 Vgl. Stefan Brieske, a.a.O. (Fn. 9), S. 17

53 Vgl. Peter Raschke, a.a.O. (Fn. 13), S. 33.

54 Vgl. Joachim Jens Hesse/ Thomas Ellwein, a.a.O. (Fn. 14), S. 162; Francois Höpflinger, a.a.O. (Fn. 10), S. 164; Jürg Martin Gabriel, Das politische System der Schweiz. Eine Staatsbürgerkunde, Bern / Stuttgart / Wien 1997, S. 50. 
spielen beschäftigt und sich nur um seine internen Sachfragen kümmert, kann morgen politisch relevant werden. " 55

Auch die Körperschaften des öffentlichen Rechts wie Kirchen, Kammern und Innungen werden häufig als öffentlich-rechtliche Interessenverbände klassifiziert. Einen „formal öffentlich-rechtlichen Status erlangen Körperschaften, wenn ihnen der Staat hoheitliche Aufgaben überträgt, sie in seinem Auftrag handeln und seiner Rechtsaufsicht unterstehen [... " ${ }^{\text {56 }}$. Die Mitgliedschaft ist hier, anders als in privatrechtlich-organisierten Verbänden, in der Regel nicht oder nur bedingt freiwillig. Zudem trifft für die kommunalen Spitzenverbände das Merkmal der Nichtstaatlichkeit nur eingeschränkt zu. Für die Kirchen gilt zudem, dass ihnen anders als anderen Interessenverbänden, durch das Grundgesetz eine besondere Stellung zugesichert wird ${ }^{57}$ und der Meinungsbildungs- und Sinngebungsprozess in der Regel nicht durch die Mitglieder vorgesehen ist. ${ }^{58}$ „Konkordate und Kirchenverträge billigen ihnen zudem Zugriffsmöglichkeiten auf öffentliche und staatliche Geltungsbereiche zu, in denen sie uneingeschränkte Verfügungsgewalt besitzen“"59, wie zum Beispiel bei der Ausbildung für Theologen.

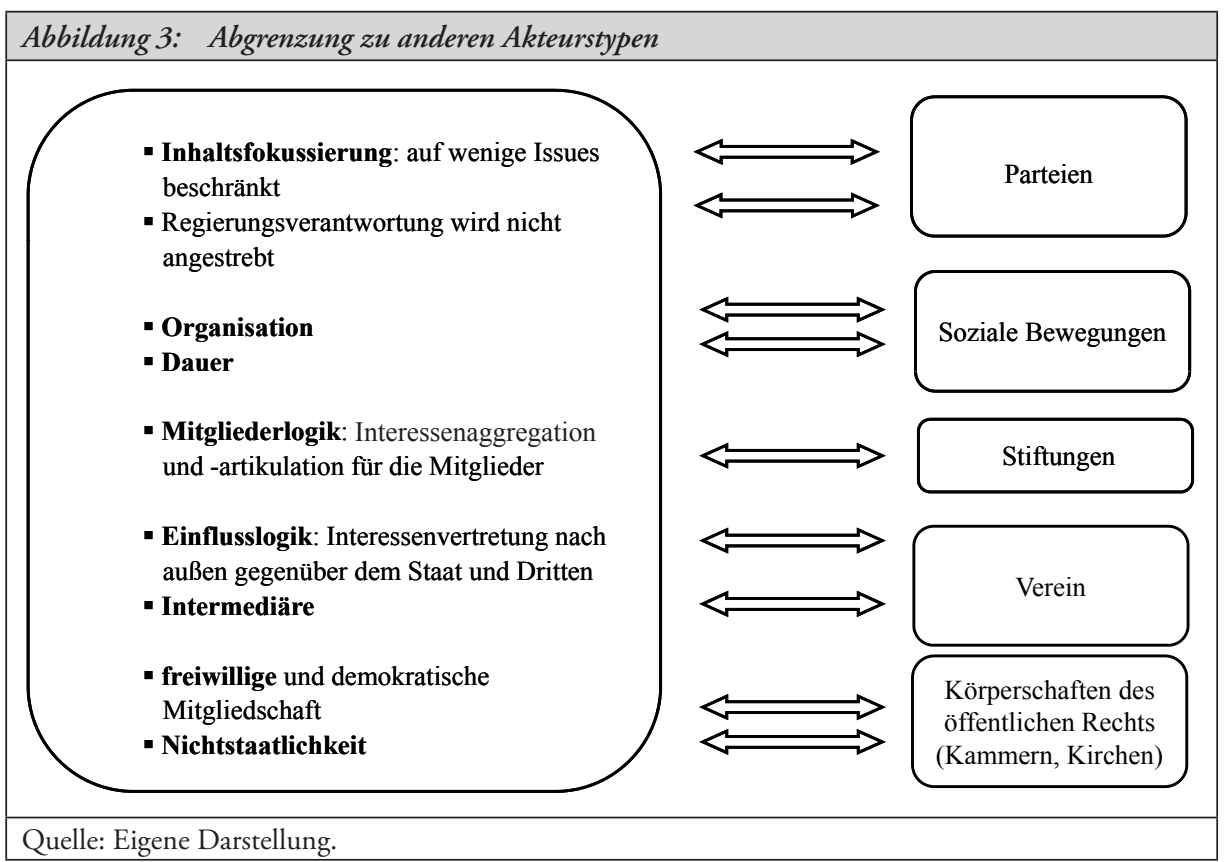

55 Sybille Laufer-Heydenreich, Die Verbände. Idee und Wirklichkeit der Verbände in der Bundesrepublik, München 1964, S. 10.

56 Werner Reutter / Peter Rütters, a.a.O. (Fn. 7), S. 78.

57 Vgl. Jürgen Weber, a.a.O. (Fn. 2), S. 155; Eckhard Jesse, Die Demokratie der Bundesrepublik Deutschland. Eine Einführung in das politische System, Berlin 1997, S. 235.

58 Vgl. Günter Triesch / Wolfgang Ockenfels, Interessenverbände in Deutschland. Ihr Einfluß in Politik, Wirtschaft und Gesellschaft, München 1995, S.168

59 Ursula Birsl, a.a.O. (Fn. 40), S. 20. 


\title{
4. Enge Definition ermöglicht eindeutige Identifikation und trennscharfe Abgrenzung von Interessenverbänden
}

Einschränkend ist für die vorliegende Meta-Analyse zu konstatieren, dass die Befunde auf einer relativ geringen Fallzahl $(n=32)$ beruhen und somit nicht als repräsentativ gelten können. Allerdings umfasst das Sample besonders relevante und aktuelle Publikationen zur Thematik, die somit die Nachteile der geringen Fallzahl nivellieren können. Die herausgearbeitete Definition erlaubt es vor allem, Interessenverbände von anderen Akteurstypen abzugrenzen. Nichtregierungsorganisationen können demzufolge auch als Interessenverband rubriziert werden. Trennscharf zu unterscheiden sind hingegen Akteure wie Stiftungen, Vereine, soziale Bewegungen und Körperschaften des öffentlichen Rechts (mit Kirchen und Kammern), die divergierenden Handlungslogiken - aufgrund der unterschiedlichen Mitgliederstrukturen, Einflussziele und staatlichen Einbindungen - folgen: Eine Kammer, die nicht um Mitglieder werben muss und mit staatlichen Aufgaben betraut ist, verfolgt andere Interessen, arbeitet mit anderen Instrumenten und innerhalb anderer Organisationsstrukturen als Umweltverbände, die um Mitglieder und politischen Einfluss werben müssen. Die hier entwickelte enggefasste Definition ermöglicht eine weitgehend zweifelsfreie Identifikation von Interessenverbänden und erleichtert deren empirische Analyse, da somit der Heterogenität und Unübersichtlichkeit des Verbandstyps durch Festlegung von Mindestcharakteristika begegnet werden kann.

\section{Die Europäische Bürgerinitiative: Inklusion neuer Akteure in die europäische Politik?}

\author{
Julian Plottka
}

Seit dem 1. April 2012 ist, rund zweieinhalb Jahre nach dem Inkrafttreten des Vertrags von Lissabon, die Verordnung über die Bürgerinitiative (EBI-Verordnung) ${ }^{1}$ gültig und ermöglicht es Unionsbürgern, gemäß Art. 11 Abs. 4 des Vertrags über die Europäische Union (EUV) eine Europäische Bürgerinitiative (EBI) anzustrengen, also die Europäische Kommission aufzufordern, einen Vorschlag für einen Rechtsakt der Union zu unterbreiten. Die dazu notwendigen eine Million Unterstützungsbekundungen müssen aus mindestens einem Viertel der Mitgliedstaaten (derzeit sieben) stammen, wobei in jedem der sieben ein Quorum zu erzielen ist, das von 4.500 in Malta und anderen Mitgliedstaaten bis zu 74.250 in Deutschland reicht. ${ }^{2}$ Nachdem eine Initiative durch die Kommission registriert wurde ${ }^{3}$,

1 Vgl. Verordnung (EU) Nr. 211/2011 des Europäischen Parlaments und des Rates vom 16. Februar 2011 über die Bürgerinitiative, in: Amtsblatt der EU, Nr. L 65 vom 11. März 2012, S. 1 - 65.

2 Vgl. Anhang I der EBI-Verordnung.

3 Zum Prüfverfahren siehe weiter unten. 